Check for updates

Cite this: Soft Matter, 2019, 15,4737

Received 27th February 2019, Accepted 16th May 2019

DOI: $10.1039 / \mathrm{c} 9 \mathrm{sm} 00428 \mathrm{a}$

rsc.li/soft-matter-journal

\title{
Light-regulated molecular diffusion in a liquid crystal network $\dagger$
}

\author{
Anping Cao, ${ }^{a}$ Roel J. H. van Raak ${ }^{a}$ and Dirk J. Broer (D) $* a b c$
}

\begin{abstract}
Photo-responsive liquid crystal polymer networks offer promising means to generate useful functional devices, but many of them focus on their mechanical response so as to generate surface features or shape change. Here, we investigate the photomechanical effect of the polymer network for molecular transport purposes. Dual wavelength illumination of an azobenzene-functionalized cholesteric liquid crystal polymer film produces excess free volume within the film, which results in an accelerated molecular diffusion through the film. Moreover, the polarization of the UV light exposure on the cholesteric network plays an important role in a remarkable enhancement of molecular diffusion. When linearly polarized UV light rotates along with the twist of the helical axis of the cholesteric polymer, excess free volume forms sequentially from the diffusion network toward the dry network in the polymer. It works in concert with the concentration gradient of the diffusant and greatly improves the diffusion through the film.
\end{abstract}

\section{Introduction}

Over many decades, work has been dedicated to developing nature-inspired stimuli-responsive polymers. ${ }^{1-3}$ These "smart" polymers can adapt to surrounding environments, convert chemical and biochemical signals into optical, electrical, thermal and mechanical signals, and vice versa.,5 These man-made materials have attracted interest for a wide range of application areas, e.g. controlled release agents, ${ }^{6,7}$ responsive coatings, ${ }^{8,9}$ and adaptive shape memory materials. ${ }^{10}$ The actuating trigger could originate from any change in the environment, such as exposure to light or heat, changes in $\mathrm{pH}$ or solvent polarity, the presence of metal ions, electric and magnetic fields or a chemical reaction. ${ }^{11,12}$

Among all stimuli, light distinguishes itself as a contactless energy source, as it can be directed from remote distances, it can be rapidly turned on or off, varied in intensity and spatially modulated across length scales. ${ }^{9,13}$ In addition, when combined with dichroic chromophores, light polarization can be utilized to modulate chemical potential. ${ }^{14}$ Motivated in part by

\footnotetext{
${ }^{a}$ Laboratory of Stimuli-Responsive Functional Materials and Devices, Department of Chemical Engineering and Chemistry, Eindhoven University of Technology, Den Dolech 2, 5612 AZ, Eindhoven, The Netherlands. E-mail: D.broer@tue.nl

${ }^{b}$ Institute for Complex Molecular Systems, Eindhoven University of Technology, Den Dolech 2, 5612 AZ, Eindhoven, The Netherlands

${ }^{c}$ SCNU-TUE Joint Lab of Devices Intergrated Responsive Materials, South China Normal University, No. 378, West Waihuan Road, Guangzhou Higher Education Mega Center, Guangzhou 510006, China

$\dagger$ Electronic supplementary information (ESI) available: Experimental methods and additional data. See DOI: 10.1039/c9sm00428a
}

these appealing properties of light, azobenzene-containing polymers (azopolymers) became one of the most commonly studied responsive materials, transducing photonic stimuli into significant alterations of specific material properties. ${ }^{15-18}$ Azobenzene molecules can undergo a reversible trans- and cis-isomerization induced by illumination. Currently, ultraviolet light irradiation induces the isomerization from transto-cis, while the reverse takes place via thermal relaxations or upon irradiation with visible light. ${ }^{19}$ Photoisomerization of azobenzene results in a large change in the molecular geometry, where the trans-isomer decreases in length between the para-carbon atoms from 10 to $6 \AA^{20}$ In turn, this results in a tremendous nanoscale force and can eventually lead to a macroscopic deformation of a polymeric system. This effect becomes enhanced when the azobenzene is embedded in a liquid crystal network where the reduction of the scalar order parameter leads to an amplification of the deformation. ${ }^{21}$ For instance, a sponge-like coating that is able to release and absorb a liquid upon exposure to light has been reported, ${ }^{22}$ where the fast release of the liquid can be induced by a macroscopic contraction of the coating caused by a trans-to-cis conversion of a copolymerized azobenzene moiety.

Previously, our group proved that continuous oscillating dynamics of the trans-to-cis isomerization of the azobenzene can boost the free volume generation in liquid crystal polymer networks. ${ }^{23}$ The excess free volume appearing in polymers is of fundamental importance for molecular transport, as it is based on the intuitively appealing picture of molecules' motion being influenced by the space around them. The study of mass transport through polymeric materials has application in a 
variety of areas such as coatings, packaging and gas separation, to name just a few. ${ }^{24-26}$ Therefore, in this work, we report the preparation of azobenzene-containing cholesteric liquid crystal networks (LCNs*) that exhibit phototunable molecular diffusion.

\section{Results and discussion}

To fabricate the $\mathrm{LCN}^{*}$, a liquid crystal mixture is prepared as shown in Fig. 1. It involves similar components as in the previous work reported by our group, ${ }^{23}$ in order to boost the generation of free volume in the cholesteric network. Notably, the non-polymerizable mesogen 3 (5CB) is present in this work as a mobile compound (porogen) to generate excess volume facilitating, after removal from the $\mathrm{LCN}^{*}$ polymer, the diffusion of other molecules. ${ }^{27}$ The mixture is aligned by shear and photopolymerzied between two glass substrates, wherein one glass was coated with rubbed polyimide (PI) and the other was treated with polyvinylacohol (PVA). The $\mathrm{LCN}^{*}$ polymer with the porogen still present has a reflection band at $\lambda=680 \mathrm{~nm}$ (Fig. 2a). After the removal of the PVA-coated substrate, mesogen 3 (porogen) is removed from the LCN by heating the $\mathrm{LCN}^{*}$ on the PI-coated glass substrate at $140{ }^{\circ} \mathrm{C}$ for $45 \mathrm{~min}$. After this procedure, the center of the reflection band shifts to $\lambda=500 \mathrm{~nm}$, revealing that the helical pitch had decreased. The cholesteric order is well preserved as shown in the scanning electron microscopy (SEM) image of a cross-section of the polymer (Fig. 2). The periodicity of the multiple parallel lines in the cross-section view represents a $\pi$ rotation in the molecular helix of a cholesteric network, as periodic alternating dark and bright areas are related to the anisotropy of the cholesteric orientation. ${ }^{28}$ As the helical structure is fixed after polymerization, the shrinkage in pitch length $(\sim 26.5 \%$ as calculated from the shift in the reflection band from $680 \mathrm{~nm}$ to $500 \mathrm{~nm}$ ) is in agreement with the amount of porogens in the liquid crystal mixture before polymerization (30 wt\%). The removal of the porogen was additionally confirmed by polarized optical microscopy (POM), i.e. in the reflection mode, the $\mathrm{LCN}^{*}$ changed color from red to green with "oil-streaks" present, which is characteristic for a cholesteric sample. Results from Fourier transform infrared spectroscopy (FT-IR) analysis indicate the complete removal of the porogen, as the characteristic signal around $2250 \mathrm{~cm}^{-1}$ for a cyano bond disappeared.

In order to investigate the mass transport properties of the $\mathrm{LCN}^{*}$ film after the removal of the porogen, mesogen 3 was drop-casted in its isotropic phase on the $\mathrm{LCN}^{*}$ film, then immediately the UV-vis transmission measurement was conducted on the sample at $38{ }^{\circ} \mathrm{C}$ in the dark, and the measurement result was collected every 6 minutes, until the transmission spectrum remained constant over time. It should be noted here that the $\mathrm{LCN}^{*}$ sample after porogen removal has a thickness of $\sim 5 \mu \mathrm{m}$, and the reflection band was around $500 \mathrm{~nm}$, i.e. the $\mathrm{LCN}^{*}$ film consists of 16 pitches, which is enough for the UV-vis transmission test. As shown in Fig. 3a, after the addition of mesogen 3 droplets on the $\mathrm{LCN}^{*}$, the reflection band around $500 \mathrm{~nm}$ gradually disappears, while the reflection band near $700 \mathrm{~nm}$ slowly appears and eventually shows a redshift. This can be attributed to the fact that mesogen 3 has gradually diffused into the flexible polymer networks, to restore the membrane structure as it was before the removal of the porogen, after which the polymer networks swell further by taking up more 3 . Taking the disappearance/appearance of the reflection band (near $500 \mathrm{~nm} / 700 \mathrm{~nm}$ ) as an indicator, the diffusion kinetics of 3 into the $\mathrm{LCN}^{*}$ in the dark can be represented by the grey curves in Fig. $3 \mathrm{~b}$ and c. The results in Fig. 3 indicate that the diffusion/sorption follows a non-Fickian pattern as can be concluded from the following features: ${ }^{29,30}$ (1) there was a boundary front, which is the main difference between Fickian
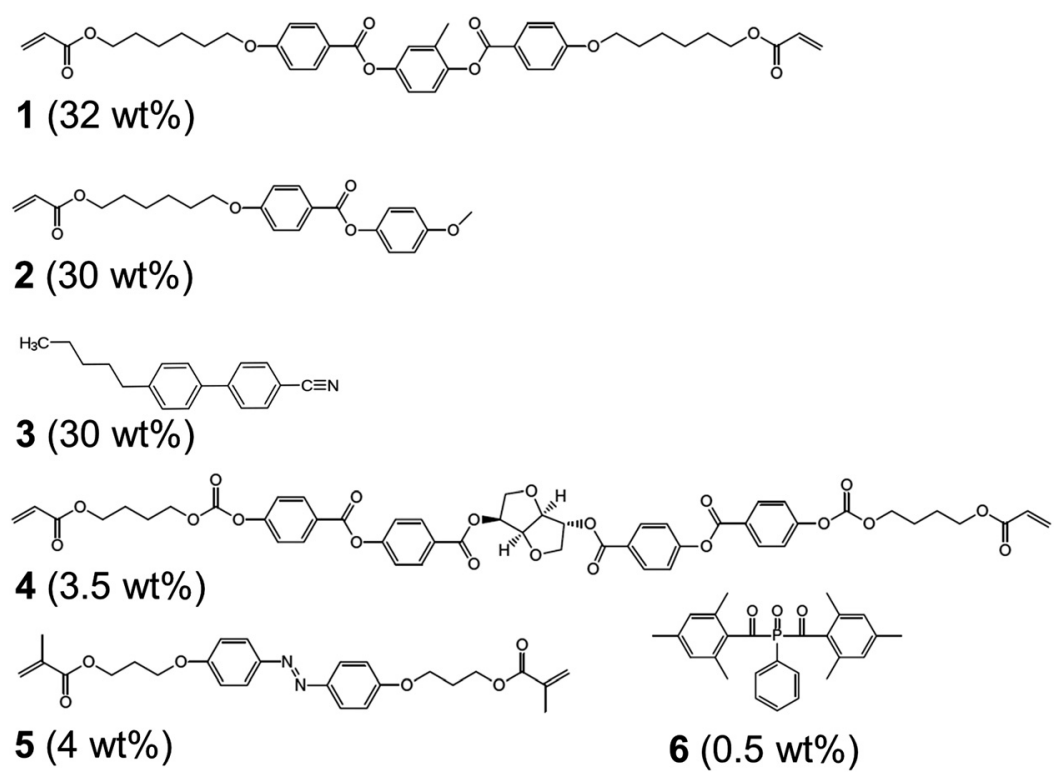

Fig. 1 Chemical structure of the liquid crystal mixture. 


\section{a}

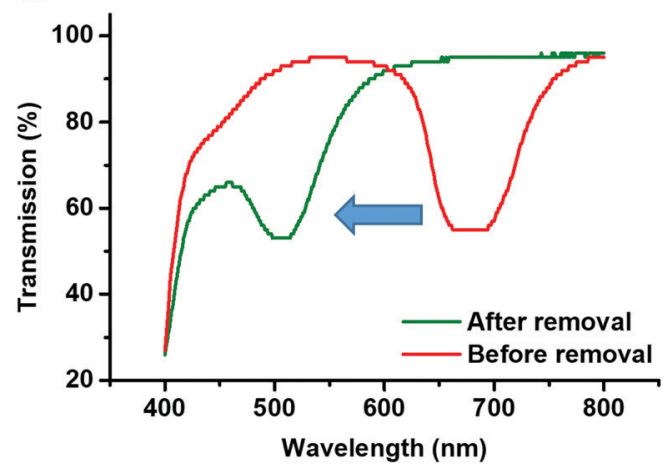

b

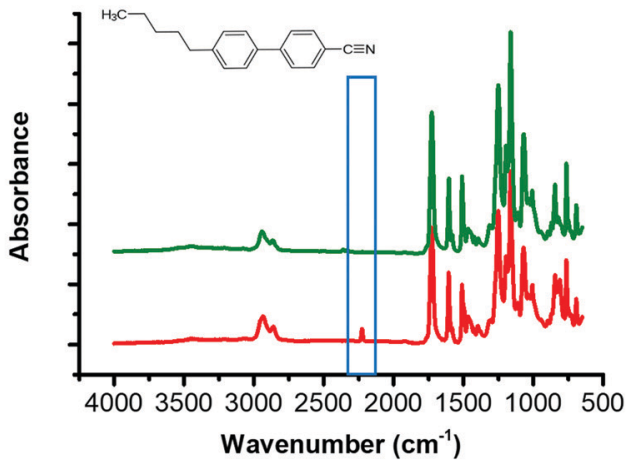

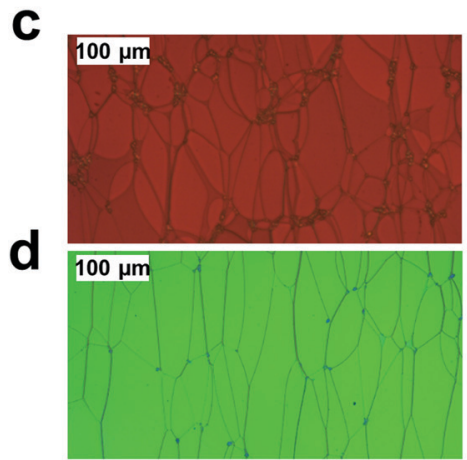

e

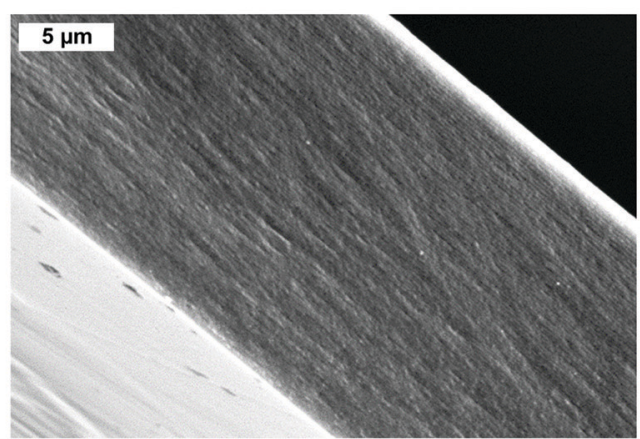

Fig. 2 (a) UV-vis transmission spectrum and (b) IR absorption spectroscopy of the LCN* film before and after the porogen removal; the LCN* film under the POM in the reflection mode before (c) and after (d) the porogen removal; (e) the SEM characterization of the cross-section of the LCN* film after the porogen removal.

a

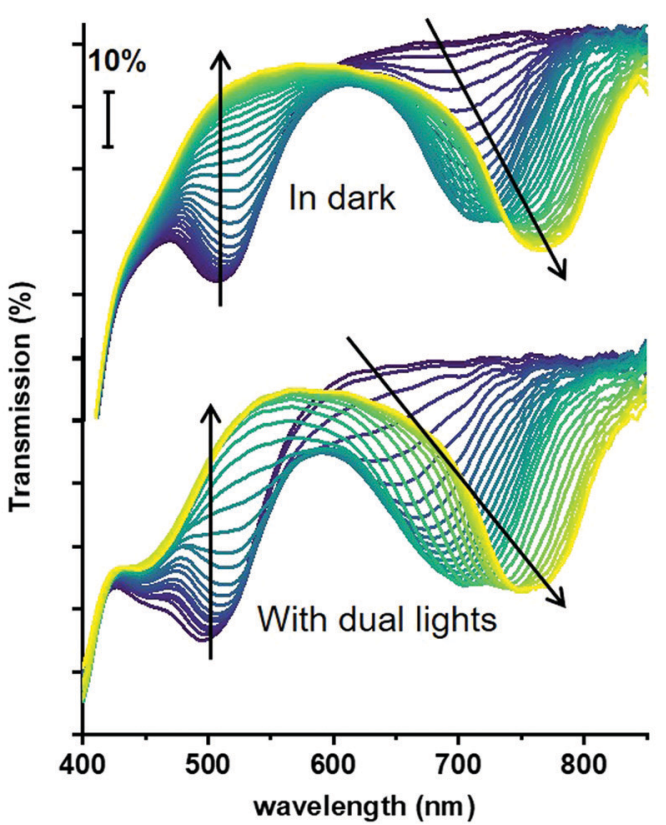

b

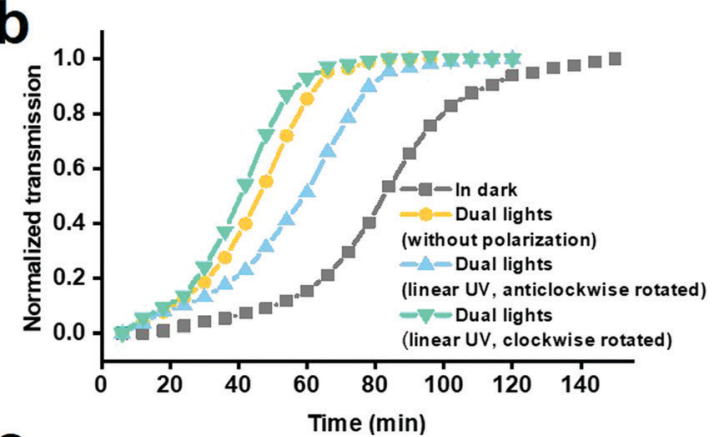

C

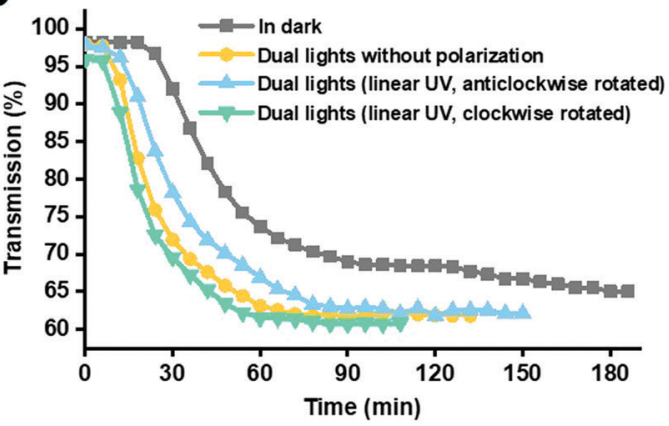

Fig. 3 UV-vis transmission test right after $5 C B$ was drop-casted on the LCN* film, (a) the raw data collected every 6 minutes; kinetics study of the $5 C B$ diffusion at different light conditions: (b) the normalized transmission of the "old" reflection band (position at $\sim 500 \mathrm{~nm}$ ) plotted against time, and (c) the transmission of the "new" reflection band (position at $\sim 700 \mathrm{~nm}$ ) over time. 
and non-Fickian diffusion. It separates the dry network from the swollen network filled with mesogen 3. In Fig. 3a, the wellseparated reflection bands at around 500 and $700 \mathrm{~nm}$ can be designated to the dry and swollen network, respectively; (2) there was an initial induction period, during which the boundary front was being established within the $\mathrm{LCN}^{*}$ film, assigned by the transmission plateau in the Z-shaped curve at the beginning of the diffusion process; (3) the boundary front moved toward the dry network of the $\mathrm{LCN}^{*}$ film with certain velocities causing the gradual increase/decrease of the transmission around $500 \mathrm{~nm} / 700 \mathrm{~nm}$ wavelength, and eventually disappeared. In the last stage of the uptake of 3 the diffusion becomes more Fickian as the reflection band at around $700 \mathrm{~nm}$ gradually shifts to higher wavelength, corresponding to the swelling of the network.

The cycle dynamics of the azobenzene with a repetitive trans-to-cis and cis-to-trans isomerization reaction can greatly enhance the formation of free volume in the $\mathrm{LCN}^{*}$, when the network is irradiated with both UV light and a small fraction of blue light, as reported earlier. ${ }^{23}$ Therefore, we adopted here dual light illumination ( $365 \mathrm{~nm}$ and $455 \mathrm{~nm}$ ) on the film to study the light response of $5 \mathrm{CB}$ diffusion into the polymer network. The intensity of UV and blue light is $100 \mathrm{~mW} \mathrm{~cm} \mathrm{~cm}^{-2}$ and $10 \mathrm{~mW} \mathrm{~cm}^{-2}$, respectively. Compared to the diffusion into the $\mathrm{LCN}^{*}$ film in the dark, illumination of the film shortens the induction period, and accelerates the diffusion, and the whole process speeds up by 28\% (130 vs. $180 \mathrm{~min}$ ), as shown in Fig. 3c (yellow vs. grey curve). Noticing that the enhancement of diffusion could be a result of the photomechanical and/or thermal effect upon illumination, the temperature of the $\mathrm{LCN}^{*}$ during the measurements with illumination has been controlled to be the same as in the test under dark conditions, as confirmed by an infrared camera. Thus, the promoted diffusion is attributed to the excess free volume formed during the dynamic isomerization of the azobenzene moieties upon dual light illumination on the $\mathrm{LCN}^{*}$ film.

Since the $\mathrm{LCN}^{*}$ matrix is a chiral system, the diffusion may also be related to the handedness of the rotation of linear polarized light during the illumination. We performed UV-vis transmission for $5 \mathrm{CB}$ diffusion into the $\mathrm{LCN}^{*}$ film under polarized light, as shown in Fig. 4a, the UV light (365 nm) is linearly polarized and the linear polarizer is mounted on a rotation stage which can rotate in a clockwise or anticlockwise direction. The blue light (455 $\mathrm{nm}$ ) is non-polarized, and keeps shining, as long as the UV light is on. The result reveals that the rotation direction of the linearly polarized UV indeed plays an important role in the diffusion process. When the rotation is in the same direction as the helical axis twisting in the
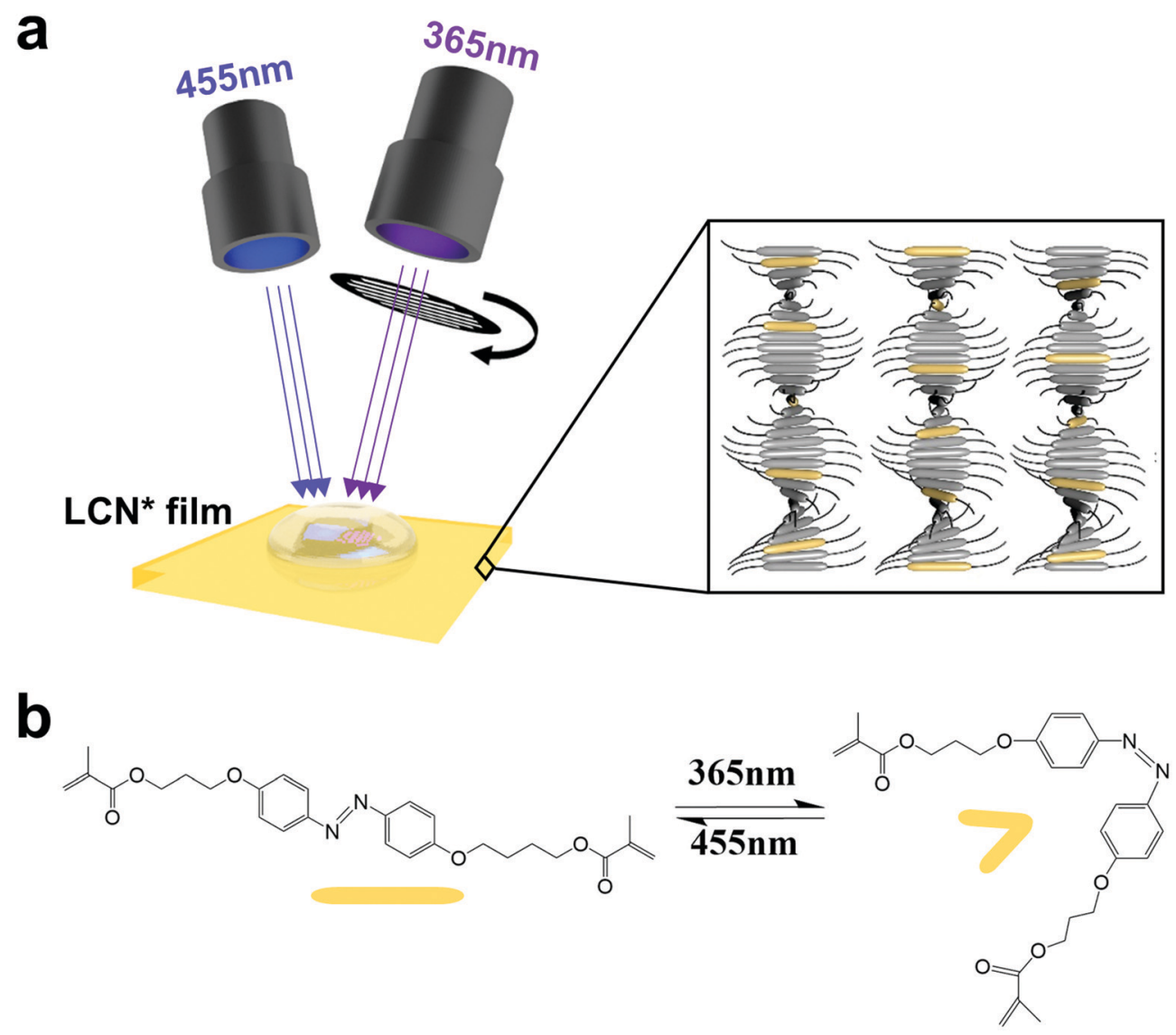

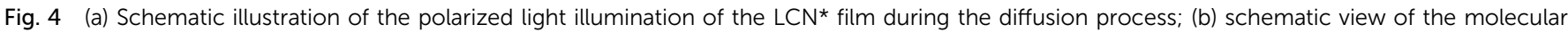

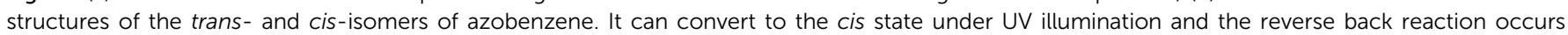
thermally or by visible light. 
right-handed $\mathrm{LCN}^{*}$ matrix (i.e. clockwise), the diffusion is enhanced by 39\% (110 vs. $180 \mathrm{~min}$ ) compared to the diffusion in the dark, as shown in Fig. 3c (green vs. grey curve). Compared to the diffusion under non-polarized light with the same light intensity on the $\mathrm{LCN}^{*}$ film, it is enhanced by $\sim 15 \%$ (110 vs. $130 \mathrm{~min}$, i.e. green vs. yellow curve in Fig. 3c). Interestingly, while the linearly polarized UV light rotated anticlockwise, i.e. an opposite direction to the handedness of the $\mathrm{LCN}^{*}$, an inhibition of $\sim 15 \%$ was observed compared to the nonpolarized case (150 vs. $130 \mathrm{~min}$, i.e. blue vs. yellow curve in Fig. 3c). This can be rationalized as, when the linearly polarized UV light rotates along the twisting direction of $\mathrm{LCN}^{*}$, the formation of excess free volume, caused by the dynamic isomerization of the azobenzene moieties (as seen in Fig. 4b), takes place around the azobenzene moieties on the top (the swollen network filled with 3 ) and gradually moves towards the bottom of the $\mathrm{LCN}^{*}$ (the dry network). The movable free volume and the concentration gradient of 3 across the $\mathrm{LCN}^{*}$ work in concert as the driving force of the diffusion. Consequently, the linearly polarized UV light rotating clockwise results in a great enhancement of the diffusion. In the case of rotation anticlockwise, the moving direction of the formation of the excess free volume is from the dry network to the swollen network in $\mathrm{LCN}^{*}$, which works against the concentration gradient. Thus, it leads to an inhibition of the diffusion compared to kinetics under non-polarized light. This elevator type of material transport is discussed in the ESI $\dagger$ and schematically shown in Fig. S2.

To further optimize the exposure conditions, the influence of the rotation speed of linearly polarized UV light on the diffusion was studied. Fig. 5a demonstrates that there is only a marginal influence of increasing the rotation speed from 2 to 25 DPS with somewhat faster diffusion at a higher rotation speed. A remaining question is whether the size of the in-diffusing molecule plays a role in the process. Thereto, liquid crystal $8 \mathrm{CB}$ diffusion into the $\mathrm{LCN}^{*}$ film at $42{ }^{\circ} \mathrm{C}$ (where $8 \mathrm{CB}$ mesogen is in an isotropic phase) was measured in the dark and with dual light illumination where the linearly polarized UV light rotated clockwise at a speed of 25 DPS. It should be noticed that the $\mathrm{LCN}^{*}$ film was prepared by using $5 \mathrm{CB}$ as a porogen, and mesogens with bigger molecular size take longer time to align within the $\mathrm{LCN}^{*}$ matrix. Nevertheless, 8CB diffusion with dual light illumination is enhanced by $\sim 50 \%$ (5 vs. 10 hours) compared to the test in the dark, as shown in Fig. 5b.

The diffusion was also tested on the $\mathrm{LCN}^{*}$ film, which was pre-treated in an ethanol solution at $50{ }^{\circ} \mathrm{C}$ for $15 \mathrm{~min}$ to extract $5 \mathrm{CB}$ before the diffusion, instead of thermal treatment to evaporate 5CB. As shown in the ESI, $\dagger$ Fig. S1, there was hardly any enhancement, comparing the diffusion in the dark to the one with dual light illumination, but overall the diffusion was faster than that in the case of using heating as pre-treatment. We speculate that the solvent extraction of 5CB may result in a relatively porous network, and the free volume created by the illumination was negligible compared to the voids caused by the removal of the porogen; thus, the photomechanical effect of the azo moieties hardly influences the diffusion. For the LCN* film with a higher cross-link density, during the preparation, the ratio between the mesogens with di-acrylate and monoacrylate was $5: 1$, a very subtle enhancement $(\sim 5 \%)$ of diffusion was found for the dual light illumination. This indicates that the rigidity of the network may play an important role, and diffusion into a film at a temperature close to its glass transition temperature would be more susceptible to the photomechanical effect. 5CB diffusion into an $\mathrm{LCN}^{*}$ polymer, which was prepared without using the porogen, has been monitored in the dark and under dual light illumination. The overall diffusion took several hours $(\sim 6 \mathrm{~h})$, and a new reflection band near $770 \mathrm{~nm}$ was formed. However, the original reflection band (near $680 \mathrm{~nm}$ ) still remained, even after the diffusion has completed. No obvious enhancement of the diffusion was found by using dual light illumination. This implies that involving the porogen in the $\mathrm{LCN}^{*}$ preparation is necessary, to create enough volume and flexibility in the network and facilitates the diffusion eventually.
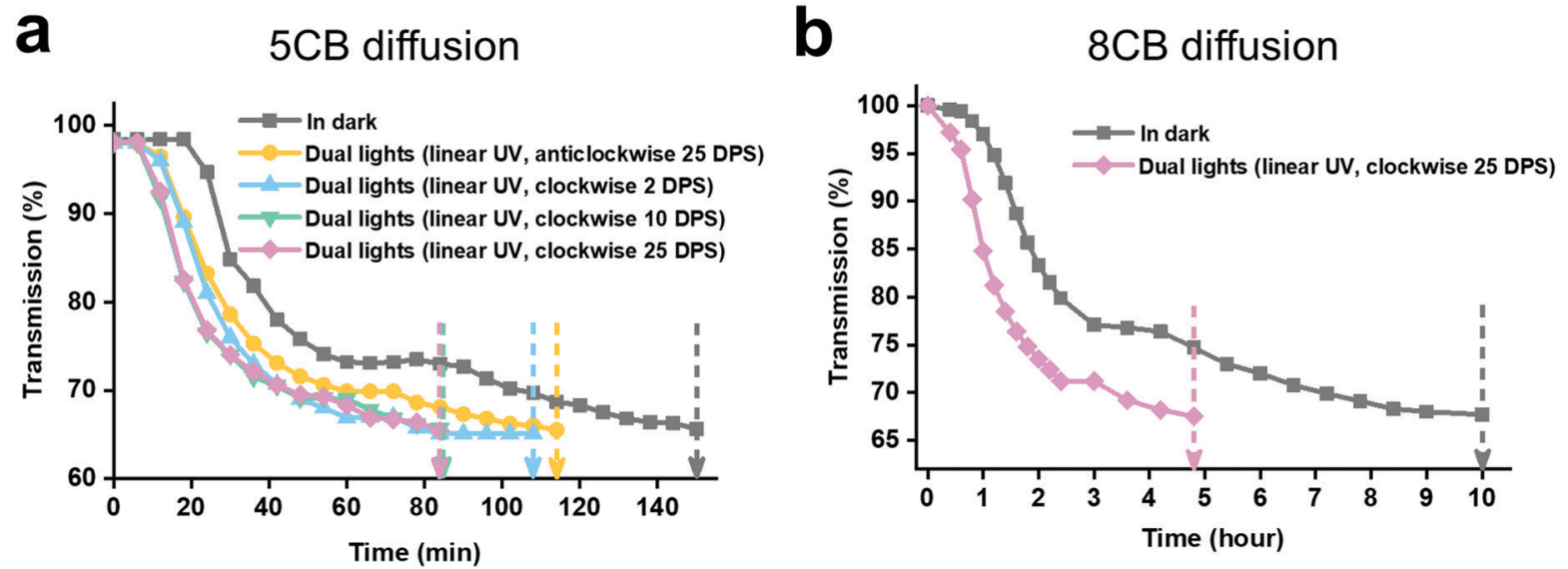

Fig. 5 Kinetics of the UV-vis transmission for (a) 5 CB diffusion in the LCN* film at different light conditions, i.e. in the dark, and with dual light where linearly polarized UV rotated in different directions and speeds; (b) 8CB diffusion in the LCN* film in the dark and with dual light illumination. DPS: degree per second. 


\section{Conclusions}

We have demonstrated that dual light (i.e. $365 \mathrm{~nm}$ and $455 \mathrm{~nm}$ ) illumination on the azobenzene-activated liquid crystal polymer improves the mesogen diffusion into the film. The accelerated diffusion is attributed to the formation of excess free volume within the network, which was caused by the oscillatory dynamics of the isomerization reaction of azobenzene. Especially when the UV light is linearly polarized and rotated along with the helical axis twisting, the formation of excess free volume moves from the swollen network filled with the diffusant towards the dry network of the polymer film, it dramatically boosts the diffusion. We have also found that the proper flexibility and rigidity of the polymer network is important for a remarkable enhancement of diffusion. In general, this study shows that the photomechanical effect of the azo-polymer is promising for the application of molecular diffusion. We anticipate that these results will open up numerous opportunities to apply the stimuli-responsive liquid crystal networks for separation and transport purposes.

\section{Conflicts of interest}

There are no conflicts to declare.

\section{Acknowledgements}

This work was financially supported by the European Research Council (Vibrate ERC, Grant 669991).

\section{References}

1 M. A. C. Stuart, W. T. S. Huck, J. Genzer, M. Müller, C. Ober, M. Stamm, G. B. Sukhorukov, I. Szleifer, V. V. Tsukruk, M. Urban, F. Winnik, S. Zauscher, I. Luzinov and S. Minko, Nat. Mater., 2010, 9, 101.

2 F. Liu and M. W. Urban, Prog. Polym. Sci., 2010, 35, 3.

3 D. Roy, J. N. Cambre and B. S. Sumerlin, Prog. Polym. Sci., 2010, 35, 278.

4 X. Z. Yan, F. Wang, B. Zheng and F. H. Huang, Chem. Soc. Rev., 2012, 41, 6042.

5 P. Theato, B. S. Sumerlin, R. K. O'Reilly and I. I. I. T. H. Epps, Chem. Soc. Rev., 2013, 42, 7055.

6 C. D. H. Alarcon, S. Pennadam and C. Alexander, Chem. Soc. Rev., 2005, 34, 276.
7 D. Schmaljohann, Adv. Drug Delivery Rev., 2006, 58, 1655.

8 I. Banerjee, R. C. Pangule and R. S. Kane, Adv. Mater., 2011, 23, 690.

9 T. J. White and D. J. Broer, Nat. Mater., 2015, 14, 1087.

10 J. S. Leng, X. Lan, Y. J. Liu and S. Y. Du, Prog. Mater. Sci., 2011, 56, 1077.

11 Intelligent Stimuli-Responsive Materials, ed. Q. Li, John Wiley \& Sons, Inc., Hoboken, NJ, 2013.

12 T. Manouras and M. Vamvakaki, Polym. Chem., 2017, 8, 74 .

13 J. J. Wie, M. R. Shankar and T. J. White, Nat. Commun., 2016, 7, 13260 .

14 M. Hendrikx, B. Sirma, A. P. H. J. Schenning, D. Liu and D. J. Broer, Adv. Mater. Interfaces, 2018, 5, 1800810.

15 A. Natansohn and P. Rochon, Chem. Rev., 2002, 102, 4139.

16 F. Ercole, T. P. Davis and R. A. Evans, Polym. Chem., 2010, 1,37 .

17 J. F. Gohy and Y. Zhao, Chem. Soc. Rev., 2013, 42, 7117.

18 F. D. Jochum and P. Theato, Chem. Soc. Rev., 2013, 42, 7468.

19 M. Hendrikx, A. P. H. J. Schenning, M. Debije and D. J. Broer, Crystals, 2017, 7, 231.

20 G. S. Kumar and D. C. Neckers, Chem. Rev., 1989, 89, 1915. 21 D. Liu, C. W. M. Bastiaansen, J. M. J. den Toonder and D. J. Broer, Angew. Chem., Int. Ed., 2012, 51, 892.

22 A. H. Gelebart, D. Liu, D. J. Mulder, K. H. J. Leunissen, J. van Gerven, A. P. H. J. Schenning and D. J. Broer, Adv. Funct. Mater., 2018, 28, 1705942.

23 D. Liu and D. J. Broer, Nat. Commun., 2015, 6, 8334.

24 S. Kim and Y. M. Lee, Prog. Polym. Sci., 2015, 43, 1.

25 K. Khosravi-Darani and D. Z. Bucci, Chem. Biochem. Eng. Q., 2015, 29, 275.

26 F. Siepmann, J. Siepmann, M. Walther, R. J. MacRae and R. Bodmeier, J. Controlled Release, 2008, 125, 1.

27 J. E. Stumpel, E. R. Gil, A. B. Spoelstra, C. W. M. Bastiaansen, D. J. Broer and A. P. H. J. Schenning, Adv. Funct. Mater., 2015, 25, 3314.

28 T. J. Bunning, D. L. Vezie, P. F. Lloyd, P. D. Haaland, E. L. Thomas and W. W. Adams, Liq. Cryst., 1994, 16, 769.

29 D. de Kee, Q. Liu and J. Hinestroza, Can. J. Chem. Eng., 2005, 83, 913.

30 M. O. Gallyamov, Eur. Phys. J. E: Soft Matter Biol. Phys., 2013, 36, 92. 